\section{Somatic symptoms in depression: evaluation of their diagnostic weight in an African setting}

\author{
G. T. OKULATE, M. O. OLAYINKA and O. B. E. JONES
}

\begin{abstract}
Background Somatic symptoms are extremely common features of depression and other mental disorders in African countries such as Nigeria, but their weight in the diagnosis of depression is not certain.
\end{abstract}

Aim To determine what weight should be assigned to these symptoms in comparison with other well-known symptoms in the diagnosis of depression.

\begin{abstract}
Method A sample of 829 persons completed the Patient Health

Questionnaire which was earlier modified by the inclusion of the somatic symptoms being studied. Using principal component analysis and a logistic regression model, the contributions of these symptoms in comparison with others were determined.
\end{abstract}

\section{Results Core depressive symptoms accounted for most of the total variance for depression. The somatic symptoms studied loaded separately from the core depressive symptoms and were not as good predictors of depression. A cognitive factor emerged as well as some somatic factors.}

\section{Conclusions Although somatic symptoms may be florid among patients with depression, they have considerably less weight than core depressive symptoms in the diagnosis of depression. The emerging cognitive factor could be similar to that described by previous authors.}

\section{Declaration of interest None.}

Somatic manifestations of depression and other common mental disorders occur across all cultures, and contribute significantly to the underrecognition of depression and anxiety in primary care practice (Goldberg, 1979; Verhaak, 1988). Somatisations have been variously described as functional, medically unexplained somatic symptoms, somatic preoccupation or worry about illness, or undue emphasis on the somatic concomitants of psychiatric disorders (Kirmayer et al, 1993). They are said to be more dominant among patients in Africa and in other developing countries (Prince, 1968; Kirmayer, 1984; Mumford et al, 1997), and even among Africans in diaspora. Brown et al (1996) showed that among depressed African Americans in the USA, the severity of somatic symptoms was higher than in their White counterparts. However, this view of a cultural preponderance is now being challenged on the basis of objective evidence (Gureje et al, 1997).

Using standard methods, several Nigerian authors have demonstrated that somatisations are extremely common features of depression (Binitie, 1975; Anumonye et al, 1979). Indeed, Ilechukwu (1991), using the Zung Depression Scale (Zung, 1965), demonstrated that somatic symptoms were more common among patients with mild to moderate depression than among those with severe depression, who tended to have more psychomotor retardation.

These authors and others (Prince, 1968; Makanjuola, 1987; Okulate \& Jones, 2002) have observed that these somatic symptoms are unique, are often related to the head in particular, and include the sensations of heat, heaviness, emptiness and skincrawling among others. Indeed, the clustering of these symptoms around the head led Makanjuola (1987) to suggest that this phenomenon might be culture-specific. Although these symptoms are ubiquitous, occurring alike in patients in primary care clinics, psychiatric clinics and traditional care settings, certain questions have yet to be clarified. Are they sine qua non symptoms of depression? If so, what weight should be assigned to them in diagnosing depression and assessing its severity among Nigerian patients, and among Africans generally?

Two issues arising from these questions form the basis of this study. First, although these unique African somatisations are well recognised, their quantification and their weightings in common mental disorders, especially depression, have not been well studied. The assessment instruments commonly used in Nigeria, the Zung Depression Scale and the Hamilton Rating Scale for Depression (Hamilton, 1961), hardly mention these unique symptoms. On the other hand, the scales developed by Binitie (1988) and Ebigbo (1982) define these symptoms well but do not attempt to relate them to diagnostic categories such as depression. Second, many African countries, particularly Nigeria, are going through huge cultural and economic changes, many of which have adverse consequences on the mental health of the people. Secondary prevention of mental illness through recognition of early symptoms is therefore a major challenge. Compared with Western societies, the relative scarcity of mental health epidemiological researchers in Africa makes the use of quick, culturally sensitive screening instruments very valuable.

\section{METHOD}

The study sample was recruited from 868 Nigerian Army personnel who reported for their year 2000 annual medical examination in our hospital (68 Nigerian Army Reference Hospital, Lagos, Nigeria). Women were excluded because they were so few. Men whose educational background would have limited their comprehension of the questionnaires and those whose responses were grossly incomplete were also excluded. Thus out of the 868 people who reported, 829 finally participated, giving a completion rate of $95.5 \%$.

The participants were given a detailed explanation of the objectives of the study, and it was emphasised that it had nothing to do with their routine annual medical examination; they were free to decline participation, and anonymity was encouraged. 
Those who consented completed the Patient Health Questionnaire (PHQ; Spitzer et al, 1999) in groups under the supervision of the two psychiatrists (G.T.O. and M.O.O.), who explained the items where necessary. In any Nigerian military population, heterogeneity of ethnicity, culture and language is the rule, because military enlistment must always reflect the wide ethnic diversity of Nigeria. Therefore, because no single language is supreme or regarded as official, translation of the PHQ into any of them was neither feasible nor desirable. In any case, only people with a minimum of secondary education (at least 10 years of school) were included. Apart from the PHQ, the participants also completed the International Index of Erectile Function (IIEF; Rosen et al, 1997), a questionnaire that assesses sexual functioning in men. This latter part of the study has been reported elsewhere (Okulate et al, 2003).

The Patient Health Questionnaire is a self-report version of the Primary Care Evaluation of Mental Disorders, developed by Spitzer et al (1999). This questionnaire has the advantage that, if well administered and cross-checked, it attempts to make definitive diagnoses. Although the authors of the PHQ believe that the diagnoses made through it are so accurate that doctors can determine and commence appropriate treatment after obtaining them, this view is not universally accepted. For this study we used the sections on somatic symptoms and depression only, excluding the panic disorder and alcohol misuse sections.

The first section of our modified questionnaire contained questions concerning the experience of somatic symptoms (without an organic basis) within the 4 weeks before the study. The experience was rated on a three-point scale 'not bothered at all', 'bothered a little', and 'bothered a lot'. In addition to the 13 symptoms provided by Spitzer et al (1999), symptoms were added by us to reflect our clinical experience and that of others (for example, Prince, 1968; Ebigbo, 1982; Ohaeri \& Odejide, 1994) in this culture. These were: heat or 'peppery' sensations in the head or body; heaviness or tension in the head; pain, emptiness or feeling of fluid within the head; and crawling sensations. The decision about which somatic symptoms to include was based on the frequencies of these symptoms as reported by these authors: for example, Ebigbo (1982) in a comparison of students and psychiatric patients found 'heat in the head' in $35.2 \%$ of students and $53 \%$ of patients, 'peppery sensation' in $11 \%$ of students and $26.8 \%$ of patients, and 'biting/crawling sensations' in $9.4 \%$ of students and $22.9 \%$ of patients.

The second section contained the classical DSM-IV criteria for depression, rated on a four-point scale (American Psychiatric Association, 1994). These criteria were: little interest or pleasure in doing things; feeling down, depressed or hopeless; trouble falling or staying asleep, or sleeping too much; feeling tired or having little energy; poor appetite; feeling bad about oneself; trouble concentrating; moving or speaking slowly; and having suicidal thoughts. This section was also expanded for our study to include difficulty in comprehension, difficulty remembering things, inability to read for long, irritability and excessive bad dreams. These additions also reflected our experience of other common symptoms of depression in this culture, using the same selection criterion as that applied for somatic symptoms. To be rated positive for depression, participants would have to score 3 or 4 'more than half of the days' or 'nearly every day' for having little interest or pleasure in doing things, or for feeling down, depressed or hopeless, as well as scoring the same on any other five symptoms of depression as laid out in the original questionnaire.

Data were analysed using the Statistical Package for the Social Sciences, version 10 (SPSS, 1998). A principal component analysis extraction adopting the varimax rotation option was used to reduce the symptoms in the somatic and the depression sections of the PHQ to a much smaller number of factors (components). Finally, being depressed or otherwise was used as an outcome variable in a logistic regression model, entering all the somatic and depressive symptoms as independent variables.

\section{RESULTS}

A total of 829 men took part in the study. Of these, $198(24 \%)$ were single, 621 $(75 \%)$ were married, $9(\mathbf{1} \%)$ were widowed and only $2(0.2 \%)$ were divorced or separated. The ages ranged between 18 years and 63 years, with a mean of 36.73 (s.d. $=9.85)$ years. Bartlett's test of sphericity gave a chi-squared score of 694.9 $(P<0.001)$, showing that the correlation matrix obtained in the factor analytic process was suitable for factor analysis.
Although only two factors (components) each explained more than $5 \%$ of the inter-item variance, eight factors had eigenvalues above 1 . Together, these eight factors accounted for $55.85 \%$ of all the variance. However, many of these factors did not appear to be clinically informative, and therefore five factors were specifically requested; these five factors together explained $46.92 \%$ of all the variance. Factor 1, with an eigenvalue of 10.03 , explained $26.40 \%$ of the variance and was labelled 'core depressive syndrome' based on the symptoms that loaded heavily on it. Table 1 shows the factor 1 loadings of these core depressive symptoms, all of which were over 0.4 . 'Hands/body shaky' was the only somatic symptom that qualified for the list. 'Excessive bad dreams' and 'unsatisfactory sleep', which were not in the original PHQ, featured prominently on the list. The other four factors had eigenvalues of $3.43,1.59,1.54$ and 1.24 respectively, and explained percentage variances of 9.04, 4.18, 4.04 and 3.26 respectively. Based on the symptoms that loaded heavily on them, factors 2 to 5 were labelled 'head somatisation', 'body somatisation', 'brain-fag syndrome' and 'somatic anxiety' respectively.

The generally low loadings of somatic symptoms (including the uniquely African ones) on factor 1 and their greater loadings on others (except factor 4) are listed in Table 2. Cognitive symptoms, which surprisingly scored low on the depressive factor, loaded heavily on factor 4 (Table 3 ). It is noteworthy that difficulty in concentrating is the only one of these cognitive symptoms that was on the original PHQ. However, it seemed to have grouped with similar symptoms on another factor (factor 4). According to the criteria suggested by the authors of the PHQ, 75 (9\%) of the people in the sample could be said to have had depression. Using a logistic regression model with depression in two categories (depressed or otherwise) as the outcome variable, all the somatic and depression symptoms were entered as independent variables. Table 4 illustrates the odds ratios and the significance levels; again, only the symptoms contributing to core depressive syndrome and back pain had odds ratios that were statistically significant. The corresponding values for the added special somatic symptoms are included in the table for comparison. Depressive feeling, loss of interest and loss of pleasure are thus clearly far better predictors of depression than are the somatic symptoms studied. 
Table I Factor loadings on factor I, core depressive syndrome $(n=829)$

\begin{tabular}{lcc}
\hline Symptom & $\begin{array}{c}\text { Frequency } \\
\text { (\%) }\end{array}$ & $\begin{array}{c}\text { Factor I loading } \\
\text { (varimax rotated) }\end{array}$ \\
\hline Loss of weight & 5.3 & 0.7 I \\
Feeling down, depressed or hopeless & 6.9 & 0.63 \\
Feeling bad about self, as if a failure & 9.7 & 0.62 \\
Sleep unsatisfactory & 7.3 & 0.59 \\
Excessive bad dreams & 6.5 & 0.59 \\
Poor appetite or overeating & 6.2 & 0.55 \\
Trouble falling asleep or staying asleep, or oversleeping & 9.7 & 0.54 \\
Feeling tired or having little energy & 9.9 & 0.54 \\
Psychomotor retardation & 3.6 & 0.53 \\
Thoughts of suicide & 1.9 & 0.49 \\
Irritability or being easily angered & 5.3 & 0.46 \\
Hands/body shaky & 3.8 & 0.46 \\
Loss of interest or pleasure & 18.5 & 0.43 \\
\hline
\end{tabular}

\section{DISCUSSION}

\section{Core depressive symptoms}

The most fundamental finding of our study is that somatic symptoms - including the unique ones described in this study - did not group with core depressive symptoms (which accounted for most of the total variance for depression). Also, they were not as good predictors of depression as were the core depressive symptoms. Thus, although somatic symptoms occur frequently in depression and other common mental disorders in Nigerian patients, our study has reiterated the views of other workers (Harding et al, 1980; Dhadphale et al, 1983) that depression would be diagnosed with certainty only if specific core symptoms were enquired about. In a similar vein, Pfeiffer (1968) studied symptoms of depression across cultures and concluded that the core symptoms of depression in other cultures were essentially the same as in Europe. To further support this point, Kirmayer et al (1993) noted that although up to $80 \%$ of patients with anxiety and depressive disorders may present exclusively with somatic complaints, the diagnoses may remain largely inaccurate if psychological symptoms are not specifically enquired about. It is noteworthy that in an international study (Gureje et al, 1997) sponsored by the World Health Organization, Ibadan, in Nigeria, was the only centre out of 15 where somatisation was found not to be significantly associated with depression.

Unlike Binitie (1975), who studied patients in a similar setting, we did not find a somatised depression factor; however, both studies agreed on the symptom of depressed mood as being central to the diagnosis of depression. The difference could be due to a number of factors. First, our study sample was exclusively male. Some authors have suggested that there is a

Table 2 Factor loadings of somatic symptoms on five factors $(n=829)$

\begin{tabular}{|c|c|c|c|c|c|c|}
\hline Symptom & Frequency (\%) & Factor I: CDS & Factor 2: HS & Factor 3: BS & Factor 4: B-FS & Factor 5: SA \\
\hline Weight loss & 5.3 & 0.71 & & & & \\
\hline Hands/body shaky & 3.8 & 0.45 & & & & \\
\hline Emptiness in head & 0.8 & 0.13 & 0.74 & & & \\
\hline Fainting spells & 1.3 & & 0.72 & & & \\
\hline Fluid inside head & 1.0 & & 0.72 & & & \\
\hline Crawling sensation, head/body & 1.3 & 0.17 & 0.70 & & & \\
\hline Shortness of breath & 1.3 & 0.26 & 0.68 & & & \\
\hline Constipation/diarrhoea & I.I & & 0.42 & & & \\
\hline Chest pain & 1.9 & 0.14 & 0.41 & & & \\
\hline Pain in head/neck & 2.3 & 0.19 & 0.29 & 0.68 & & \\
\hline Back pain & 3.6 & 0.16 & 0.17 & 0.66 & & \\
\hline Waist pain & 4.0 & & 0.15 & 0.59 & & \\
\hline Heaviness in head/neck & 1.3 & 0.25 & 0.35 & 0.58 & & \\
\hline Tension in head/neck & I.I & 0.20 & 0.49 & 0.56 & & \\
\hline Pain in arms/legs & 5.1 & 0.14 & & 0.49 & & \\
\hline Headache & 3.2 & 0.22 & & 0.34 & & \\
\hline Heart beating fast & 2.3 & 0.24 & 0.27 & 0.13 & & 0.56 \\
\hline Heat/peppery sensations & 1.8 & 0.21 & 0.29 & 0.21 & & 0.53 \\
\hline Nausea/indigestion & 1.9 & & 0.37 & & & 0.49 \\
\hline Dizziness & 3.0 & & & & & $0.4 I$ \\
\hline
\end{tabular}

B-FS, brain-fag syndrome; BS, body somatisation; CDS, core depressive syndrome; HS, head somatisation; SA, somatic anxiety.

Empty cells denote very low loadings. 
Table 3 Cognitive symptom loadings on factors I and $4(n=829)$

\begin{tabular}{|c|c|c|c|}
\hline Symptom & Frequency (\%) & Factor I loading & Factor 4 loading \\
\hline Difficulty in remembering & 6.0 & 0.24 & 0.66 \\
\hline Slowed mental operation & 2.4 & 0.19 & 0.64 \\
\hline Difficulty in comprehension & 2.8 & 0.21 & 0.61 \\
\hline Inability to read for long & 11.7 & 0.25 & 0.48 \\
\hline Trouble concentrating on activities such as reading or watching television & 12.7 & 0.26 & 0.48 \\
\hline
\end{tabular}

Factor I, core depressive syndrome; Factor 4, brain-fag syndrome.

greater tendency for women to use somatic language to express psychological discomfort compared with men (Hobbs et al, 1984). Also, there could have been some change in the mode of presentation of depression over the 33 years since Binitie conmethods using more efficient computer software could have made a difference.

Two symptoms deserve some comment. Excessive bad dreams and unsatisfactory sleep were not part of the original PHQ but featured prominently in the core depressive syndrome factor. These symptoms might be culturally determined and therefore might not have featured in the North American population among whom the original PHQ was developed. Unsatisfactory sleep featured in addition to trouble falling asleep or remaining asleep on this factor, yet they do not necessarily have the same meaning to patients.

\section{Cognitive factor}

Cognitive symptoms scored low on the depressive factor, but loaded heavily on ducted his study. Last, improved statistical

factor 4. It is noteworthy that difficulty in concentrating is the only one of these cognitive symptoms that was on the original PHQ (and DSM-IV) criteria for depression. It must have loaded fairly highly with core depressive symptoms to justify inclusion during the development of these instruments. However, in our study it seemed to be grouped with similar symptoms on another factor (factor 4). In the apparent absence of dementia or delirium, this grouping of cognitive symptoms might be similar to the brain-fag syndrome described by Prince (1985) and Morakinyo (1985). It could be argued that all the cognitive symptoms assessed in this study reflect difficulty with concentration. However, we observed that there were variations in the frequency of reporting the symptoms, varying from $2.8 \%$ for difficulty in comprehension to $12.7 \%$ for difficulty concentrating (see Table 3).

Although brain-fag syndrome occurs also among those who are not necessarily involved in intellectual work, other symptoms apart from cognitive ones are

Table 4 Odds ratios (for various symptoms) with depression as outcome variable in a logistic regression model

\begin{tabular}{lccc}
\hline Symptom & Odds ratio & $95 \% \mathrm{Cl}$ & $P$ \\
\hline Loss of interest & 128.43 & $4.09-4037.08$ & 0.006 \\
Feeling depressed & 31.22 & $2.12-458.8$ & 0.012 \\
Trouble concentrating & 14.42 & $1.68-123.53$ & 0.015 \\
Poor appetite & 6.93 & $1.04-46.09$ & 0.045 \\
Tiredness & 5.55 & $0.87-35.62$ & 0.071 \\
Feeling bad about self & 4.94 & $0.91-26.95$ & 0.065, NS \\
Back pain & 2.68 & $1.12-6.43$ & 0.028 \\
Crawling sensations & 14.957 & $0.03-7114.66$ & 0.39, NS \\
Pain in the head & 12.48 & $0.09-1565.96$ & 0.306, NS \\
Fluid in the head & 5.68 & $0.00-8.4$ & 0.837, NS \\
Tension in the head & 1.67 & $0.03-95.79$ & 0.804, NS \\
Heaviness in the head & 0.086 & $0.00-54.57$ & 0.456, NS \\
Emptiness in the head & 0.080 & $0.00-1810.75$ & 0.622, NS \\
Heat in the head and body & 0.014 & $0.00-6.21$ & 0.170, NS \\
\hline
\end{tabular}

required to make the diagnosis. These are essentially somatic symptoms. Thus, our attempt to justify this syndrome based on the factor 4 loadings will require further study.

\section{Instrument development}

Our finding concerning the weight of somatic symptoms in the diagnosis of depression has an important implication with respect to the development of culturally sensitive instruments for depression in Nigeria. It can be inferred that if such instruments include somatic symptoms, the latter might have to be considered low down on the list of items and only be regarded as secondary symptoms. It is pertinent to note that in the development of such a questionnaire for use in Zimbabwe, Patel et al (1997) included many somatic items at the commencement of the procedure, but following multivariate analysis only one somatic symptom made it into the final instrument. On the other hand, Mumford et al (1997), in an epidemiological survey in a rural part of Pakistan, observed that if the screening instrument used had not included several somatic symptoms, many cases of neurosis would have been missed.

\section{Somatisation factor}

If, as our study suggests, somatisations do not have strong diagnostic weight in depression, will they have more weight in generalised anxiety disorders, panic disorder, obsessive-compulsive disorder and somatisation disorder? This may well be true, as revealed by their strong loadings on factors 2, 3 and 5. However, this requires further study, in view of the fact that these disorders (except somatisation disorder), if clearly diagnosed, usually show good response to chemotherapeutic intervention. Furthermore, the large gathering of somatic symptoms around the head in factor 2 may reflect a type of somatisation disorder peculiar to Africans. Indeed, 
Ebigbo (1982) suspected that such a phenomenon could exist and, like other workers, wondered how appropriate Western international classification models such as the DSM could be for Africans, particularly when considering neurotic disorders.

\section{Limitations}

The limitation of this study resulting from the use of an all-male sample has been mentioned. There is also a need to sound a note of caution concerning the sensitivity and specificity of the PHQ in this particular study. Although anonymity was encouraged and the participants were reassured, the fact that the instrument was administered just prior to their annual fitness medical examination might have introduced some false negatives. These were people who mostly wished to continue with military service. On the other hand, the absence of additional clinical or structured interviews might have produced some false positives. Nevertheless, it is noteworthy that even among false positives in an epidemiological study, mental disorders at sub-syndromal levels and even other types of mental disorder are usually extremely common (Leon $e t a l$, 1997). These other disorders quite often cause functional impairment and deserve psychiatric attention in their own right.

\section{Significance of somatic symptoms}

Despite their florid presence in people with depression, somatic symptoms should be considered as secondary to the core depressive symptoms in arriving at the diagnosis. Also, rating scales for depression in African cultures should continue to emphasise the core depressive symptoms, as in other cultures, in addition to the somatic symptoms. Nevertheless, from a dynamic perspective, the symbolic significance of these peculiar somatic symptoms should not be underrated, despite our findings, especially if viewed in their sociocultural context. In such circumstances their meanings would be best clarified through explorative dynamic interventions.

\section{REFERENCES}

American Psychiatric Association (1994) Diagnostic and Statistical Manual of Mental Disorders (4th edn) (DSM-IV). Washington, DC: APA

Anumonye, A., llechukwu, S. T. C. \& Adaranijo, H (1979) Patterns of depression in a Nigerian megalopolis. African Journal of Psychiatry, 5, 67-74.

\section{CLINICAL IMPLICATIONS}

Somatic symptoms account for only a little of the total variance for depression, hence diagnosis continues to rely on the presence of the core depressive symptoms.

- The low weight of the somatic symptoms has implications for the development of depression rating instruments in this setting.

The emergence of a cognitive factor reopens the issue of a culture-specific syndrome previously described by others.

\section{LIMITATIONS}

Only men were included in the study sample.

- The problem of false negatives and false positives for depression on the Patient Health Questionnaire requires further consideration.

- The possible contribution of organic disorders to the presence of somatic symptoms also requires investigation.

G. T. OKULATE, MBChB, FMCPsych(Nig), FWACP, M. O. OLAYINKA, MBBS, FWACP, O. B. E. JONES, BSc(Psych), MSc(Psych), 68 Nigerian Army Reference Hospital, Yaba, Lagos, Nigeria

Correspondence: Dr G.T. Okulate, PO Box 8869, Shomolu, Lagos State, Nigeria.

E-mail: okulateus@yahoo.com

(First received I2 June 2003, final revision II November 2003, accepted 2 December 2003)

Binitie, A. (1975) A factor-analytic study of depression across cultures (African and European). British Journal of Psychiatry, 127, 559-563.

Binitie, A. (1988) Outstanding contributions to Nigerian psychiatry. Nigerian Journal of Psychiatry, $\mathbf{I}$ $145-154$.

\section{Brown, C., Schulberg, H. C. \& Madonia, M. J. (1996)} Clinical presentations of major depression by African Americans and Whites in primary medical care practice. Journal of Affective Disorders, 4I, |8|-|9|.

Dhadphale, M., Ellison, R. H. \& Griffin, L. (1983) The frequency of psychiatric disorders among patients attending semi-urban and rural general out-patient clinics in Kenya. British Journal of Psychiatry, 142 379-383.

Ebigbo, P. O. (1982) Developing a culture specific (Nigeria) screening scale of somatic complaints indicating psychiatric disturbances. Culture, Medicine and Psychiatry, 6, 29-43.

Goldberg, D. (1979) Detection and assessment of emotional disorders in a primary care setting. International Journal of Mental Health, 8, 30-48.

Gureje, O., Simon, G. E., Ustun, T. B., et al (1997) Somatization in cross-cultural perspective: a World Health Organization study in primary care. American Journal of Psychiatry, 154, 989-995.

Hamilton, M. (1961) Development of a rating scale for primary depressive illness. British Journal of Social and Clinical Psychology, 6, 278-296.
Harding, T.W., Arangode, M.V., Beltazar, J., et al (1980) Mental disorders in primary health care: a study of their frequency and diagnosis in four developing countries. Psychological Medicine, 10, 231-24l.

Hobbs, P., Ballinger, C., Greenwood, B., et al (1984) Factor analysis and validation of the General Health Questionnaire in men: a general practice survey. British Journal of Psychiatry, 144, 270-275.

llechukwu, S. T. C. (1991) Psychiatry in Africa: specia problems and unique features. Transcultural Psychiatric Research Review, 28, 169-218.

Kirmayer, L. (1984) Culture, affect and somatization II. Transcultural Psychiatric Research Review, 21, 237-262.

Kirmayer, L. J., Robbins, J. M., Dworkind, M., et al (1993) Somatization and the recognition of depression and anxiety in primary care. American Journal of Psychiatry, I50, 734-741.

Leon, A. C., Portera, L., Olfson, M., et al (1997) False positive results: a challenge for psychiatric screening in primary care. American Journal of Psychiatry, I54, $1462-1464$.

Makanjuola, R. O. A. (1987) 'Ode-ori'. A culture bound disorder with prominent somatic features in Yoruba-Nigerian patients. Acta Psychiatrica Scandinavica, 75, 23I-236

Morakinyo, O. (1985) The brain-fag syndrome in Nigeria: cognitive deficits in an illness associated with study. British Journal of Psychiatry, 146, 209-210. 
Mumford, D. B., Saeed, K., Ahmad, I., et al (1997) Stress and psychiatric disorder in rural Punjab: a community survey. British Journal of Psychiatry, 170 $473-478$.

Ohaeri, J. U. \& Odejide, O. A. (1994) Somatization symptoms among patients using primary health care facilities in a rural community in Nigeria. American Journal of Psychiatry, 15I, 28-31.

Okulate, G.T. \& Jones, O. B. E. (2002) Two depression rating instruments in Nigerian patients. Nigerian Postgraduate Medical Journal, 9, 74-78.

Okulate, G. T., Olayinka, M. O. \& Jones, O. B. E.

(2003) Erectile dysfunction. Prevalence and relationship to depression, alcohol abuse and panic disorder. General Hospital Psychiatry, 25, 209-213.
Patel, V., Simunyu, E., Gwanzura, F., et al (1997) The Shona Symptom Questionnaire: the development of an indigenous measure of common mental disorders in Harare. Acta Psychiatrica Scandinavica, 95, 469-475.

Pfeiffer, W. (1968) The symptomatology of depression viewed transculturally. Transcultural Psychiatric Research Review, 5, 121 .

Prince, R. H. (1968) The changing picture of depressive syndromes in Africa: is it fact or diagnostic fashion? Canadian Journal of African Studies, I, 177-192.

Prince, R. H. (1985) The concept of culture bound syndromes. Anorexia nervosa and brain-fag. Social Science and Medicine, 2I, 197-203.

Rosen, R.C., Riley, A., Wagner, G., et al (1997) The International Index of Erectile Function (IIEF): a multidimensional scale for assessment of erectile dysfunction. Urology, 49, 822-830.

Spitzer, R. L., Kroenke, K. \& Williams, J. B.W. (1999) Validation and utility of a self-report version of PRIMEMD. The PHQ primary care study. JAMA, 282, 17371744.

SPSS (1998) SPSS for Windows, version 10. Chicago, IL: SPSS Inc.

Verhaak, P. F. M. (1988) Detection of psychological complaints by general practitioners. Medical Care, $\mathbf{2 6}$ 1009-1020.

Zung, W. W. K. (1965) A self-rating depression scale. Archives of General Psychiatry, 12, 63-70. 